\title{
ANÁlISIS COMPARATIVO DE PROFESIO- NISTAS, PROFESORES Y ESTUDIANTES MEXICANOS EN CUANTO A SUS VALORES $Y$ SUS IMPLICACIONES RESPECTO AL TRABAJO
}

Comparative analysis of professionals, teachers and Mexican students in terms of their values and their implications for the work

\section{María Teresa de la Garza Carranza* Eugenio Guzmán Soria** Alfonso Cervantes Maldonado ${ }^{* * *}$}

Resumen: El presente artículo de investigación realiza una evaluación de 600 personas (estudiantes, profesores y profesionales) para identificar sus principales valores de acuerdo al instrumento desarrollado por Schwartz. Para lograr que el análisis sea confiable, se realizó un análisis de confiabilidad y validez. Los resultados indican que los valores prioritarios para estos grupos son la

María Teresa de la Garza Carranza. Doctora en Ciencias con Especialidad en Ciencias Administrativas por el Instituto Politécnico Nacional. Actualmente es profesora e investigadora del Instituto Tecnológico de Celaya (México). Email: teresa.garza@itcelaya.edu.mx

** Eugenio Guzmán Soria. Es profesor investigador del Postgrado en Administración en el Departamento de Ciencias Económico Administrativas del Instituto Tecnológico de Celaya (México). Email: Eugenio.guzman@itcelaya.edu.mx

*** Alfonso Cervantes Maldonado. Realizó estudios de Ingeniería Industrial en el Instituto Tecnológico de León y maestría en Ingeniería de la Calidad en la Universidad Iberoamericana. Email: acervantes@delasalle.edu.mx 
Maria Teresa de la Garza Carranza, Eugenio Guzmán Soria, Alfonso Cervantes Maldonado

"auto-trascendencia" y el valor de "conservar". El proyecto aporta parámetros confiables sobre los valores de este instrumento en México.

Palabras clave: Análisis comparativo, profesionista, valores en el trabajo, cuestionario de Schwartz, México.

Abstract: This research paper assesses 600 people (students, teachers and professionals) to identify their key values according to the instrument developed by Schwartz. To ensure that the analysis is reliable, an analysis of reliability and validity was performed. The results indicate that the priority values for these groups are "self-transcendence" and the value "preserve". The project provides reliable parameters on the values of this instrument in Mexico.

Keywords: Comparative analysis, professional, work values, Schwartz questionnaire, Mexico.

\section{Introducción}

En este artículo se hace un análisis comparativo de profesionistas, profesores y estudiantes mexicanos en cuanto a sus valores y sus implicaciones respecto al trabajo. El objetivo de este estudio es en una primera instancia identificar rasgos diferentes de tres grupos de estudio (profesores, profesionistas y estudiantes) en México utilizando un instrumento que ha probado su validez en repetidas mediciones en el mundo. Algunos de los resultados muestran claramente que no todas las culturas ni los individuos son iguales y que existen diferencias con relación a su escala de valores. 
La exposición se desarrollará en dos momentos, el primer momento alude al marco referencial y la metodología que se empleó, en el segundo momento se discuten los resultados.

\section{Marco teórico}

Para comparar los valores culturales de los países, generalmente se realizan promedios estadísticos de los reportes obtenidos de los cuestionarios de las personas encuestadas o entrevistadas. El procedimiento generalmente utilizado es a través de encuestas auto-administradas y este es el enfoque que se ha seguido en los estudios internaciones para determinar los rasgos característicos de las culturas nacionales (Hofstede, 1980; House et al., 2004, etc.). Pero generalmente, los miembros de la sociedad tienen rasgos característicos y este enfoque estaría justificado si los miembros de una sociedad tuvieran los mismos valores centrales o sistemas normativos (Schwartz, 2014).

Actualmente existen dos tendencias de los rasgos característicos de análisis de las diferentes culturas del mundo. Uno es el enfoque genérico donde se han tomado a las culturas como bloques (América Latina, Asiático, Anglosajón, etc.) y un enfoque más reciente es el de estudiar de manera individual las características nacionales. En el primer enfoque han existido estudios internacionales donde se caracterizan a las culturas por rasgos principales (Hofstede, 1980; House et al., 2002 y Schwartz, 2006); el segundo enfoque consiste en remarcar que cada cultura es diferente y no puede ser agrupada en bloques generales ya que inclusive podrían existir diferencias de valores entre una ciudad y otra. Este enfoque ha sido explicado por Jones, Felps y Gregory (2007), Mizzau y Montanari (2008) y recientemente por Sasaki y Yoshikawa (2013). La importancia que un enfoque intra-organizacional tiene para la ventaja competitiva de las organizaciones ha sido establecida por Mizzau y Montanari (2008), Ralston et al. (2013), entre otros. 
Maria Teresa de la Garza Carranza, Eugenio Guzmán Soria, Alfonso Cervantes Maldonado

De acuerdo con Sasaki y Yoshikawa (2013), existen cuatro razones principales para estudiar la cultura como una unicidad en lugar de utilizar un enfoque generalista. En primer lugar, asumir una homogeneidad cultural implica no observar diferencias entre las diferentes naciones; es decir, esto implicaría por ejemplo, que no hubiera diferencias entre los colombianos y los gerentes colombianos. En segundo lugar, los rasgos diferenciales entre los diferentes grupos de un país no son apreciados por los enfoques genéricos; es decir posiblemente existan más factores que intervienen en el comportamiento de las personas como puede ser la educación, la posición jerárquica en la organización, etc. En tercer lugar, existen variables moderadoras debido por ejemplo al tipo de organización (pública o privada) o bien al giro de la misma (manufactura o servicios) por ejemplo. Finalmente, las características culturales son movibles en el tiempo; algunos estudios sugieren (Ralston, 2008) que las culturas nacionales cambian sus características en función de variables económicas. El objetivo de este estudio es en una primera instancia identificar rasgos diferentes de tres grupos de estudio (profesores, profesionistas y estudiantes) en México utilizando un instrumento que ha probado su validez en repetidas mediciones en el mundo.

En referencia a los valores, encontramos diversas posturas que analizan el concepto bajo diferentes puntos de vista. Komblit (1994) analiza los valores desde el punto de vista sociológico, definiéndolos como estructuras cognitivas y complejas que implican dimensiones evaluativas y conductuales. Mientras que la postura psicológica, define a los valores como concepciones de lo deseable que influyen en las formas en que la gente evalúa eventos y elige vías de acción. Los valores sirven de base tanto para actitudes, como para conductas, orienta las actitudes, el actuar de individuos y grupos (Cayón y Pérez, 2008). Por su parte, Rockeach (1973) plantea la idea de un sistema de valores al que define como una organización aprendida de principios y reglas que guían al individuo a seleccionar conductas frente a una situación; y a su vez, desarrolla un sistema 
de medición de la jerarquía de valores que plantea. En tanto que el modelo planteado por Schwartz (1999) constituye una estructura del sistema de valores que se relaciona con las conductas del individuo, pero es claro que la mayoría coinciden en que se encuentran directamente relacionados con la conducta. Schwartz (1992) también definió los valores como metas deseables, que traspasan situaciones, que varían en importancia y que sirven como principios en la vida de una persona o entidad social.

Sin embargo también existe una relación entre los valores de una persona y la cultura de un país. De acuerdo con Hofstede (2001), la cultura es un programa mental que cada persona tiene y es estable en el tiempo de tal manera que lleva a esa persona a comportarse de manera igual bajo situaciones similares. Entonces, cada persona tiene una programación en parte compartida y en parte individual. La parte compartida se deberá a los valores universales y colectivos y la parte individual a la región, religión o bien a la influencia familiar. A continuación se muestra un diagrama (figura N. ${ }^{\circ} 1$ ) con la relación entre los valores y el comportamiento.

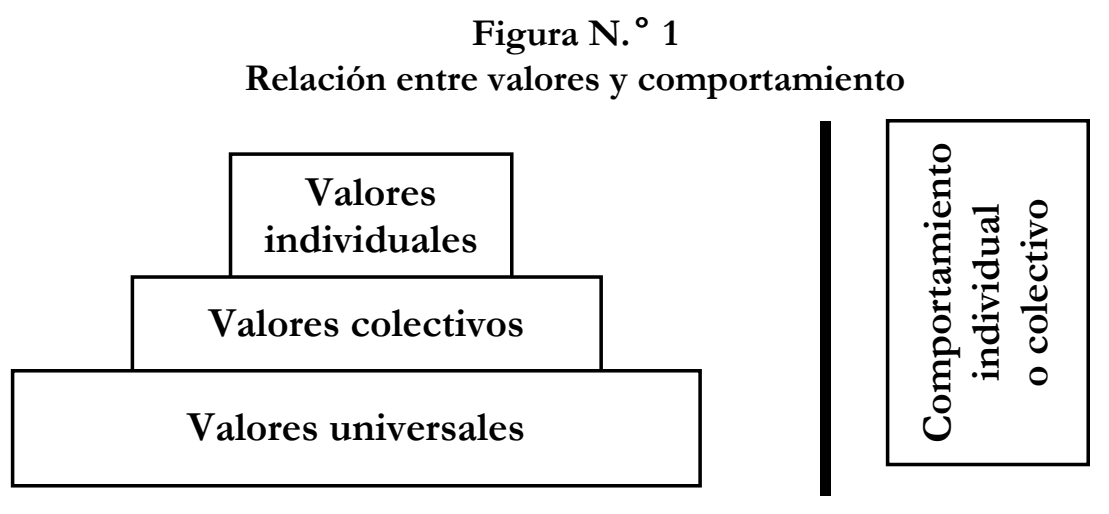

Es importante comprender cuáles son los valores ya que de acuerdo a lo que Schwartz (2014) postula, estos expresan la forma en que las instituciones son organizadas, sus políticas y las prácticas de día 
María Teresa de la Garza Carranza, Eugenio Guzmán Soria, Alfonso Cervantes Maldonado

a día de manera explícita o implícita llevadas a cabo por una sociedad. Estos también explican los sistemas de competencia económica, la forma de hacer confrontaciones en los sistemas legales, la forma de crianza hacia el logro de metas de los niños, etc.

Los valores pueden ser definidos de muchas maneras ya que el término ha sido usado para referirse a una gran cantidad de conceptos incluyendo intereses, placeres, gustos, preferencias, obligaciones morales, necesidades, aversiones y atracciones (Williams, 1979). Apoyándose en la teoría de la jerarquía de valores, Maslow (1954) los define como guías u orientaciones que los seres humanos utilizan para la satisfacción de necesidades, los cuales se encuentran organizados en jerarquías. Según Cayón y Pérez (2008) un sistema claro de valores produce a la persona bienestar, pues le hace más fácil tomar decisiones y elegir su camino y, por el contrario, si el sistema de valores es indefinido produce conflictos, malestar e incertidumbre al momento de tomar decisiones.

Lo descrito en el párrafo anterior guía la discusión, alrededor de la aplicación de los valores a las conductas, ya que las decisiones que tomamos son en virtud de nuestro sistema de valores; pero como afirma Burns (1978), las acciones también son influidas por agentes externos, que no solo pueden llevar al individuo a actuar en contra de su sistema de valores. En ocasiones estos agentes externos son incluso capaces de modificar el sistema de valores. Como un ejemplo de lo anterior, puede pensarse en el caso de los líderes que generan comportamiento socialmente no deseados, como el de Adolfo Hitler, caso que nos invita a reflexionar respecto la influencia de los valores de estos agentes externos sobre los subordinados, pues al considerar los valores como guías de actuación, implica también que definan en buena parte el estilo de liderazgo ejercido.

Como ejemplo de la aplicabilidad de los valores, podemos decir que la parte observable de los sistemas de valores de los líderes son sus actitudes y conductas consecuencia de sus sistemas de 
valores como ya se comentó, y como consecuencia de estas, la influencia en las actitudes y acciones de sus seguidores, influencia que de prolongarse en el tiempo, llevarían a un cambio permanente en el sistema de valores de los seguidores.

De acuerdo con Schwartz y Bilsky (1987), los valores tienen las siguientes características:

- Los valores son creencias. Sin embargo no son objetivos, ni ideas fijas.

- Los valores se refieren a metas deseables y a modelos de conducta que promueven esas metas.

- Los valores trascienden acciones específicas y situaciones.

- Los valores guían la selección o evaluación de comportamiento, personas y eventos.

- Los valores son ordenados por importancia relativa de unos con otros.

El modelo planteado por Schwartz (1994), diseña un sistema que puede agruparse para conformar categorías en cuanto a las acciones personales y colectivas, así como en cuanto a los tipos de liderazgo. En este modelo se mencionan diez tipos de valores motivacionales, personales y universales:

1. Poder: búsqueda de estatus y prestigio social, dominio de las personas y los recursos (poder social, autonomía, riqueza).

2. Logro: búsqueda del éxito personal, tendencia a ser competitivo dentro de lo socialmente aceptable (éxito, capaz, ambicioso, influyente).

3. Hedonismo: búsqueda del placer y la gratificación personal, y disfrute de la vida (placer, disfrutar de la vida).

4. Estimulación: tendiente a lo novedoso e interesado en los desafíos (retos, vida variada, vida excitante). 
Maria Teresa de la Garza Carranza, Eugenio Guzmán Soria, Alfonso Cervantes Maldonado

5. Autodirección: independiente, libre pensador, pensamiento creativo, tener metas propias.

6. Universalismo: búsqueda de la justicia social, tolerancia y preocupación por el medio ambiente (de mente abierta, sabiduría, justicia social, igualdad, un mundo de paz, un mundo de belleza, unidad con la naturaleza).

7. Benevolencia: búsqueda del bienestar de las personas, la honestidad y sociabilidad (servicial, honrado, saber perdonar, leal, responsable)

8. Tradición: Respeto y compromiso por las costumbres y la cultura tradicional, así como con la religiosidad (humilde, aceptar mi parte de la vida, devoto, respeto por la tradición, moderado).

9. Conformidad: Restricción de acciones, autocontrol de inclinaciones e impulsos que puedan trastornar o de dañar otros y violar expectativas o normas sociales (Educado, obediente, autodisciplina, honrar a padres y mayores).

10. Seguridad: interesan la seguridad, armonía y estabilidad social y personal (seguridad familiar, seguridad nacional, orden social, limpio, reciprocidad de favores).

De acuerdo con Schwartz (1996), los valores mostrados entre paréntesis en el párrafo anterior son universales ya que han sido validados en diferentes culturas; existen otros que no son universales como por ejemplo el auto respeto, salud, sentido de pertinencia, etc. Para llegar a estos valores Schwartz (1994) explica que se utilizó el siguiente razonamiento: "Para hacer frente a la realidad en el contexto social, los grupos y los individuos transforman de manera cognitiva las necesidades inherentes de la existencia humana y la expresan en el lenguaje de valores específicos con los cuales puede comunicarse. Específicamente, los valores representan, en forma de metas conscientes, respuestas a tres requerimientos 
universales que todos los individuos y sociedades deben enfrentar: las necesidades como organismos biológicos, requisitos para una coordinación social integrada y requisitos para el buen funcionamiento y sobrevivencia de los grupos" (p. 21). De este concepto básico se derivaron los diez valores de la tipología; donde, por ejemplo, el valor "poder" está relacionado con las necesidades de integración social y de grupos. De igual modo, el valor "hedonismo" está relacionado con la necesidad orgánica, etc.

Estos valores se agrupan en cuatro categorías, las que son de interés personal en función del interés colectivo "auto-trascendencia", las que dan prioridad a los intereses propios "autopromoción" o "auto-mejora", aquellos que aprecian la seguridad y el orden "conservación" y las que prefieren la independencia de acciones y pensamientos sobre la "apertura al cambio". De acuerdo con la teoría de Schwartz, los valores aparecen en un diagrama circular de forma dinámica. Los valores contiguos dentro del ciclo son compatibles mientras que los valores opuestos son contradictorios y pueden generar conflicto, por ejemplo, el valor de logro está en conflicto con la benevolencia: las personas que buscan el logro podrían tener dificultades si también desean ser serviciales y mejorar el bienestar de otros. Sin embargo, ser servicial puede ser compatible con aceptar las costumbres culturales y religiosas de acuerdo a la tradición por lo que estos valores van en la misma dirección. Véase al respecto la figura $\mathrm{N}{ }^{\circ} 2$.

Si se toma en consideración lo anterior, el objetivo de esta investigación es identificar primeramente si el instrumento desarrollado por Schwartz tiene validez y confiabilidad estadística en México y, en un segundo término, evaluar si el instrumento tiene el mismo significado para estudiantes, profesores y maestros mexicanos y determinar cuáles son las prioridades de estos tres grupos con relación a la apertura al cambio, la auto-mejora, la auto-trascendencia y la búsqueda de la conservación. 
Maria Teresa de la Garza Carranza, Eugenio Guzmán Soria, Alfonso Cervantes Maldonado

Figura N. ${ }^{\circ} 2$

Relación de los valores propuestos por Schwartz

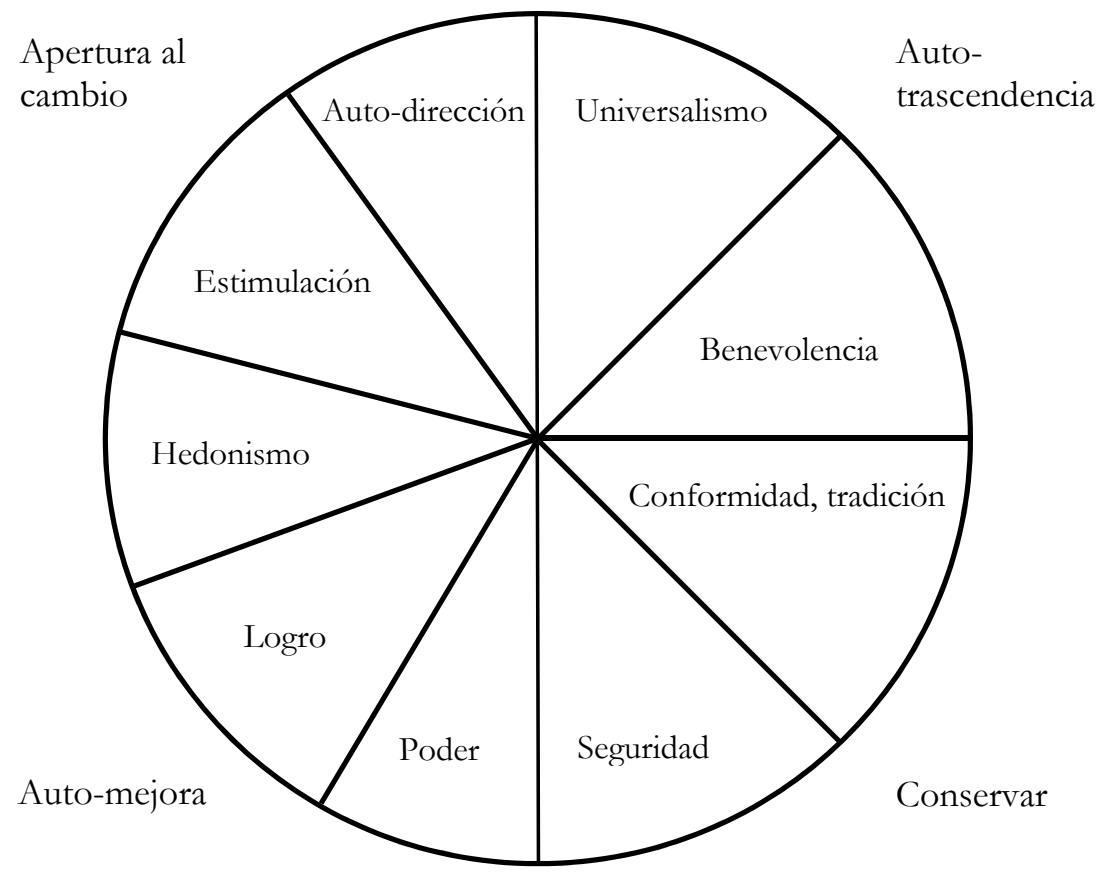

\section{Metodología}

La preparación para el proyecto consistió en hacer una traducción del inglés al español del cuestionario desarrollado por Schwartz y Bilsky (1987). Además se realizó nuevamente la traducción del español al inglés para tener consistencia en la traducción. Se levantaron pruebas piloto y se utilizó una escala Likert de (1 a 7) tomando en cuenta que estos dos valores extremos deben de ser colocados al valor que menos es importante para la persona y ante el que más lo es. El resto de los números $(0,1,2,3,4,5$ y 6) se indicaban al entrevistado que se buscaba utilizar todos los números. 
Para realizar el proyecto, primeramente se levantó información de 200 profesores de educación primaria, secundaria y preparatoria: 200 estudiantes de licenciatura que estuvieran inscritos en cualquier área de negocios y 200 profesionistas en el Estado de Guanajuato. La información se recabó durante el año de 2013. Se buscó que hubiera en cada uno de los 3 grupos 50\% de mujeres y 50\% de hombres. Como primer paso se hicieron las pruebas respectivas de validez y confiabilidad de constructo tomando en consideración 44 de los ítems desarrollados por Schwartz; se realizaron pruebas de análisis factorial y alfa de Crobach para cada uno de los indicadores del valor propuesto. Como segundo paso, se analizaron los resultados de cada uno de los valores para encontrar aquellos que tuvieran mayor importancia para los grupos estudiados.

\section{Resultados}

Primeramente se calculó el Alfa de Cronbach es un coeficiente que sirve para medir la fiabilidad de una escala de medida y es una media de las correlaciones entre las variables que forman parte de la escala. Los resultados del alfa de Cronbach para el instrumento de Schwartz para los profesores es de $\alpha=0.903$, para los estudiantes es de $\alpha=0.931$ y para los profesionales es de $\alpha=0.894$. Esto muestra que el cuestionario es fiable. Para medir la confiabilidad, se realizaron análisis factoriales sobre los constructos de cada uno de los valores probando las tres poblaciones de estudio. El análisis factorial es una técnica de reducción de datos que sirve para encontrar grupos homogéneos de variables a partir de un conjunto numeroso de ítems; el análisis también indica el porcentaje de varianza explicada por cada uno de los factores de una variable. Los resultados factoriales mostraron lo siguiente para cada uno de los tres grupos analizados: 
Maria Teresa de la Garza Carranza, Eugenio Guzmán Soria, Alfonso Cervantes Maldonado

Tabla N. ${ }^{\circ} 1$

Resultados comparativos del valor "poder"

\begin{tabular}{|l|c|c|c|}
\hline \multicolumn{1}{|c|}{ Ítem } & $\begin{array}{c}\text { Carga de ítems } \\
\text { profesionales }\end{array}$ & $\begin{array}{c}\text { Carga de ítems } \\
\text { profesores }\end{array}$ & $\begin{array}{c}\text { Carga de ítems } \\
\text { estudiantes }\end{array}$ \\
\hline Poder Social & 0.827 & 0.758 & 0.743 \\
\hline Riqueza & 0.786 & 0.611 & 0.801 \\
\hline Autoridad & 0.514 & 0.698 & 0.769 \\
\hline Varianza explicada & $52 \%$ & $48 \%$ & $59 \%$ \\
\hline
\end{tabular}

Como se muestra en la tabla $\mathrm{N} .^{\circ} 1$, para el valor "poder" la varianza explicada por los tres grupos es de alrededor del 50\% y en los tres grupos la carga factorial es de más de 0.50 , podemos concluir que este valor presenta confiabilidad y validez estadística para los tres grupos estudiados.

Tabla N. ${ }^{\circ} 2$

Resultados comparativos del valor "logro"

\begin{tabular}{|l|c|c|c|}
\hline \multicolumn{1}{|c|}{ Ítem } & $\begin{array}{c}\text { Carga de ítems } \\
\text { profesionales }\end{array}$ & $\begin{array}{c}\text { Carga de ítems } \\
\text { profesores }\end{array}$ & $\begin{array}{c}\text { Carga de ítems } \\
\text { estudiantes }\end{array}$ \\
\hline Exitoso & 0.711 & 0.760 & 0.725 \\
\hline Ambicioso & 0.705 & 0.675 & 0.570 \\
\hline Capaz & 0.687 & 0.756 & 0.737 \\
\hline Influyente & 0.649 & 0.501 & 0.652 \\
\hline Varianza explicada & $47 \%$ & $46 \%$ & $45 \%$ \\
\hline
\end{tabular}

En la tabla N. ${ }^{\circ} 2$ se muestran los resultados del valor logro, los ítems muestran una buena carga factorial y la varianza explicada es de alrededor del $46 \%$ con lo que se demuestra una buena confiabilidad y validez estadística. 
Análisis comparativo de profesionistas, profesores y estudiantes mexicanos en cuanto a sus valores y sus implicaciones respecto al trabajo

Tabla N. ${ }^{\circ} 3$

Resultados comparativos del valor "hedonismo"

\begin{tabular}{|l|c|c|c|}
\hline \multicolumn{1}{|c|}{ Ítem } & $\begin{array}{c}\text { Carga de ítems } \\
\text { profesionales }\end{array}$ & $\begin{array}{c}\text { Carga de ítems } \\
\text { profesores }\end{array}$ & $\begin{array}{c}\text { Carga de ítems } \\
\text { estudiantes }\end{array}$ \\
\hline Disfrutar de la vida & 0.816 & 0.829 & 0.816 \\
\hline Placer & 0.816 & 0.829 & 0.816 \\
\hline Varianza explicada & $67 \%$ & $69 \%$ & $66 \%$ \\
\hline
\end{tabular}

Aunque el valor hedonismo está representado únicamente por dos ítems, se muestra una buena carga factorial y la varianza explicada es alta como se indica en la tabla $\mathrm{N} .^{\circ} 3$.

\section{Tabla N. ${ }^{\circ} 4$}

Resultados comparativos del valor "estimulación"

\begin{tabular}{|l|c|c|c|}
\hline \multicolumn{1}{|c|}{ Ítem } & $\begin{array}{c}\text { Carga de ítems } \\
\text { profesionales }\end{array}$ & $\begin{array}{c}\text { Carga de ítems } \\
\text { profesores }\end{array}$ & $\begin{array}{c}\text { Carga de ítems } \\
\text { estudiantes }\end{array}$ \\
\hline Vida excitante & 0.811 & 0.697 & 0.795 \\
\hline Atrevido & 0.757 & 0.717 & 0.795 \\
\hline Vida variada & 0.714 & 0.709 & 0.756 \\
\hline Varianza explicada & $58 \%$ & $50 \%$ & $55 \%$ \\
\hline
\end{tabular}

El análisis del valor estimulación aplicado a los tres grupos muestra una buena carga factorial y la varianza explicada oscila alrededor del 55\%. Se presentan resultados estadísticos confiables.

La tabla N. ${ }^{\circ} 5$ muestra que para el grupo de profesionistas, el valor de "auto-dirección" no todos los ítems contribuyen a explicar este valor y se muestran dos grupos de respuestas unos asociados con metas propias, ser independiente y curioso y otras relacionadas con la libertad y la creatividad. En este sentido hay que ser precavido en la aplicación de este cuestionario a profesionales. Para el grupo de estudiantes y de profesores todos los ítems contribuyen a explicar el valor pero la varianza explicada es representativa pero apenas aceptable. 
Maria Teresa de la Garza Carranza, Eugenio Guzmán Soria, Alfonso Cervantes Maldonado

Tabla N. ${ }^{\circ} 5$

Resultados comparativos del valor "auto-dirección"

\begin{tabular}{|l|c|c|c|c|c|c|}
\hline \multirow{2}{*}{\multicolumn{1}{|c|}{ Ítem }} & \multicolumn{2}{|c|}{$\begin{array}{c}\text { Carga de ítems } \\
\text { profesionales }\end{array}$} & \multicolumn{2}{c|}{$\begin{array}{c}\text { Carga de ítems } \\
\text { profesores }\end{array}$} & \multicolumn{2}{c|}{$\begin{array}{c}\text { Carga de ítems } \\
\text { estudiantes }\end{array}$} \\
\cline { 2 - 7 } & Factor 1 & Factor 2 & Factor 1 & Factor 2 & Factor 1 & Factor 2 \\
\hline Metas propias & 0.828 & & 0.758 & & 0.721 & \\
\hline Independiente & 0.721 & & 0.719 & & 0.702 & \\
\hline Curioso & 0.616 & & 0.635 & & 0.591 & \\
\hline Libertad & & 0.812 & 0.345 & & 0.576 & \\
\hline Creatividad & & 0.640 & 0.580 & & 0.678 & \\
\hline Varianza explicada & & $59 \%$ & $37 \%$ & & $43 \%$ & \\
\hline
\end{tabular}

Tabla N. ${ }^{\circ} 6$

Resultados comparativos del valor "universalismo"

\begin{tabular}{|l|c|c|c|c|c|c|}
\hline \multirow{2}{*}{ Ítem } & \multicolumn{2}{|c|}{$\begin{array}{c}\text { Carga de ítems } \\
\text { profesionales }\end{array}$} & \multicolumn{2}{c|}{$\begin{array}{c}\text { Carga de ítems } \\
\text { profesores }\end{array}$} & \multicolumn{2}{c|}{$\begin{array}{c}\text { Carga de ítems } \\
\text { estudiantes }\end{array}$} \\
\cline { 2 - 7 } & Factor 1 & Factor 2 & Factor 1 & Factor 2 & Factor 1 & Factor 2 \\
\hline Mundo de belleza & 0.803 & & & 0.773 & 0.830 & \\
\hline $\begin{array}{l}\text { Protección del } \\
\text { medio ambiente }\end{array}$ & 0.737 & & 0.578 & & 0.542 & \\
\hline Justicia social & 0.697 & & 0.567 & & 0.552 & \\
\hline Sabiduría & 0.672 & & & 0.662 & 0.753 & \\
\hline $\begin{array}{l}\text { Unidad con la } \\
\text { naturaleza }\end{array}$ & 0.461 & & 0.694 & & 0.589 & \\
\hline Igualdad & & 0.789 & 0.569 & & 0.594 & \\
\hline Mente abierta & & 0.594 & & 0.709 & & 0.784 \\
\hline Mundo de paz & & 0.529 & 0.803 & & & 0.712 \\
\hline Varianza explicada & & $52 \%$ & & $54 \%$ & & $55 \%$ \\
\hline
\end{tabular}


En la tabla N. ${ }^{\circ} 6$ se muestra que el valor "universalismo" está compuesto por dos grupos de ítems. El grupo de profesionistas es semejante al grupo de profesionistas, pero esto no sucede así con el grupo de profesores. La varianza explicada es alta; pero en resumen hay que tener cuidado cuando se aplique este instrumento ya que presenta resultados diferentes en los 3 grupos estudiados.

\section{Tabla N. ${ }^{\circ} 7$}

Resultados comparativos del valor "benevolencia"

\begin{tabular}{|l|c|c|c|}
\hline \multicolumn{1}{|c|}{ Ítem } & $\begin{array}{c}\text { Carga de ítems } \\
\text { profesionales }\end{array}$ & $\begin{array}{c}\text { Carga de ítems } \\
\text { profesores }\end{array}$ & $\begin{array}{c}\text { Carga de ítems } \\
\text { estudiantes }\end{array}$ \\
\hline Perdón & 0.736 & 0.801 & 0.661 \\
\hline Responsabilidad & 0.671 & 0.746 & 0.732 \\
\hline Leal & 0.647 & 0.691 & 0.639 \\
\hline Honrado & 0.638 & 0.474 & 0.672 \\
\hline Servicial & 0.378 & 0.605 & 0.665 \\
\hline Varianza explicada & $39 \%$ & $45 \%$ & $45 \%$ \\
\hline
\end{tabular}

El valor "benevolencia" presenta cargas factoriales adecuadas y una varianza explicada con alrededor de $40 \%$ en los tres grupos, como se muestra en la tabla N. ${ }^{\circ}$.

Tabla N. ${ }^{\circ} 8$

Resultados comparativos del valor "conformidad"

\begin{tabular}{|l|c|c|c|}
\hline Ítem & $\begin{array}{c}\text { Carga de ítems } \\
\text { profesionales }\end{array}$ & $\begin{array}{c}\text { Carga de ítems } \\
\text { profesores }\end{array}$ & $\begin{array}{c}\text { Carga de ítems } \\
\text { estudiantes }\end{array}$ \\
\hline Autodisciplina & 0.731 & 0.552 & 0.575 \\
\hline Educado & 0.663 & 0.709 & 0.638 \\
\hline Honrado & 0.612 & 0.445 & 0.732 \\
\hline Obediencia & 0.573 & 0.732 & 0.702 \\
\hline Varianza explicada & $42 \%$ & $38 \%$ & $44 \%$ \\
\hline
\end{tabular}

El valor "conformidad" presenta resultados muy semejantes en los tres grupos estudiados tal y como se muestra en la tabla N. ${ }^{\circ} 8$. 
Maria Teresa de la Garza Carranza, Eugenio Guzmán Soria, Alfonso Cervantes Maldonado

Tabla N. ${ }^{\circ} 9$

Resultados comparativos del valor "seguridad"

\begin{tabular}{|l|c|c|c|c|c|l|}
\hline \multirow{2}{*}{\multicolumn{1}{|c|}{ Ítem }} & \multicolumn{2}{c|}{$\begin{array}{c}\text { Carga de ítems } \\
\text { profesionales }\end{array}$} & \multicolumn{2}{c|}{$\begin{array}{c}\text { Carga de ítems } \\
\text { profesores }\end{array}$} & \multicolumn{2}{c|}{$\begin{array}{c}\text { Carga de ítems } \\
\text { estudiantes }\end{array}$} \\
\cline { 2 - 7 } & Factor 1 & Factor 2 & Factor 1 & Factor 2 & Factor 1 & Factor 2 \\
\hline Orden social & 0.745 & & 0.747 & & 0.496 & \\
\hline $\begin{array}{l}\text { Reciprocidad } \\
\text { de favores }\end{array}$ & 0.716 & & 0.591 & & 0.648 & \\
\hline Limpio & & 0.792 & 0,487 & & 0.640 & \\
\hline Seguridad familiar & & 0.575 & 0.68 & & 0.746 & \\
\hline Seguridad nacional & & 0.553 & 0.655 & & 0.688 & \\
\hline Varianza explicada & & $55.6 \%$ & $41 \%$ & & $42 \%$ & \\
\hline
\end{tabular}

El valor seguridad presenta resultados semejantes para profesores y estudiantes mientras que para los profesionistas no. En el caso de los profesionistas, se ven dos factores involucrados: uno relacionado con el orden social y la reciprocidad de favores y otro relacionado con la limpieza, la seguridad nacional y familiar. En los 3 grupos de estudio la varianza explicada es alta como se muestra en la tabla N. ${ }^{\circ} 9$.

Tabla N. ${ }^{\circ} 10$

Resultados comparativos del valor "tradición"

\begin{tabular}{|l|c|c|c|}
\hline Ítem & $\begin{array}{c}\text { Carga de ítems } \\
\text { profesionales }\end{array}$ & $\begin{array}{c}\text { Carga de ítems } \\
\text { profesores }\end{array}$ & $\begin{array}{c}\text { Carga de ítems } \\
\text { estudiantes }\end{array}$ \\
\hline Moderado & 0.657 & 0.712 & 0.656 \\
\hline Humilde & 0.637 & 0.498 & 0.682 \\
\hline $\begin{array}{l}\text { Aceptar la posición } \\
\text { en la vida }\end{array}$ & 0.625 & 0.546 & 0.668 \\
\hline Devoto & 0.571 & 0.629 & 0.569 \\
\hline Tradición & 0.404 & 0.753 & 0.682 \\
\hline Varianza explicada & $34 \%$ & $40 \%$ & $42 \%$ \\
\hline
\end{tabular}


Los resultados comparativos del valor "tradición" muestran resultados muy similares en los tres grupos estudiados por lo que se demuestra su validez y confiabilidad como se detalla en la tabla N. ${ }^{\circ} 10$.

Además se realizó una tabla comparativa con cada uno de los valores para indicar cuáles eran los promedios de cada uno de ellos. Como se muestra en la gráfica 1 los valores más apreciados por los tres grupos estudiados son los que están relacionados con los valores de "autodirección" y de "benevolencia". En el caso de los resultados del valor "universalismo", los resultados deben ser tomados con cuidado ya que estos no son confiables como se mostró con anterioridad.

\section{Gráfica $\mathbf{N}^{\circ}{ }^{1}$}

\section{Resultados comparativos de los tres grupos estudiados}

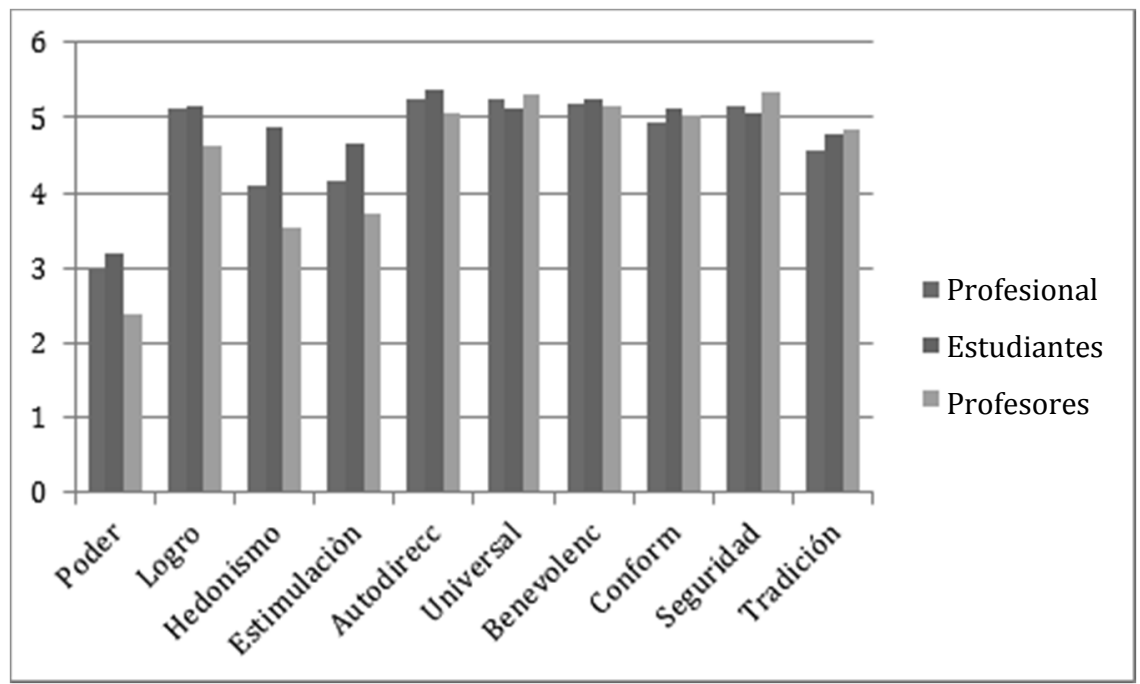

Finalmente, basándonos en la figura $\mathrm{N}{ }^{\circ} 1$, se tomaron los valores de "benevolencia" y "universalismo" para calcular la autotrascendencia; los valores de "seguridad", "conformidad" y "tradición" para calcular los valores de conservación y los valores de "logro", "poder" y "hedonismo" para calcular la auto-mejora 
Maria Teresa de la Garza Carranza, Eugenio Guzmán Soria, Alfonso Cervantes Maldonado

y, finalmente, los valores de "autodirección", "estimulación” y "hedonismo" para el cálculo de la apertura al cambio. Los resultados se muestran en la gráfica N. ${ }^{\circ} 2$.

Gráfica $\mathrm{N}^{\circ} 2$

Cuadro comparativo de valores complementarios

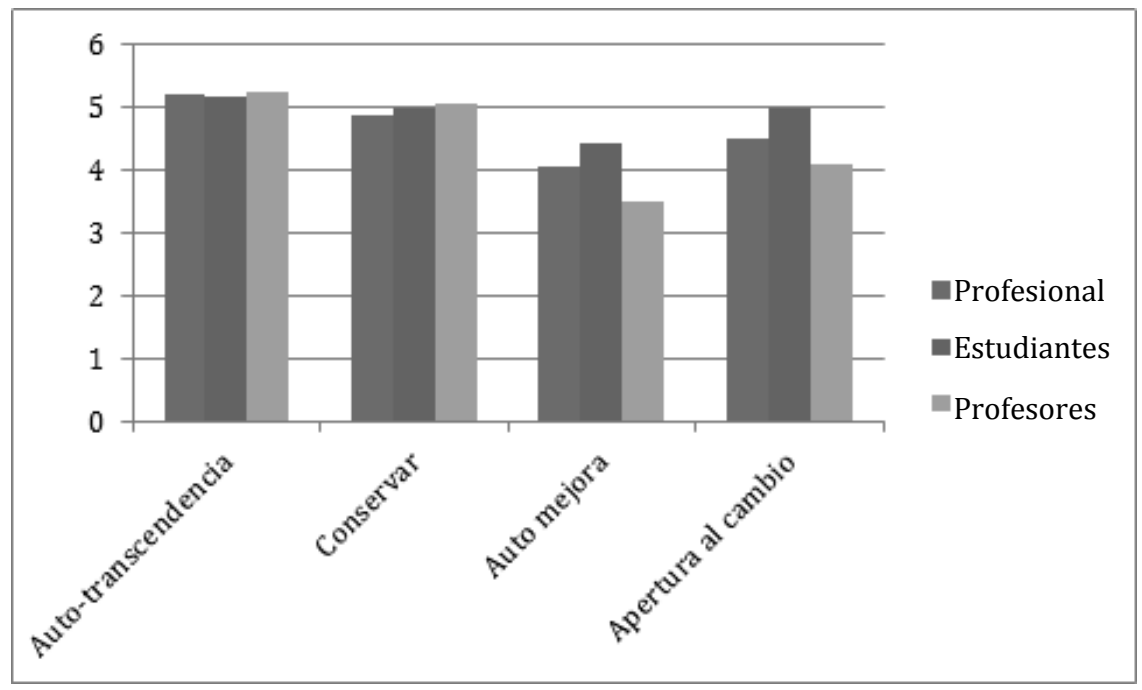

Como se muestra en la gráfica, los valores complementarios de "auto-trascendencia" y "conservar" predominan sobre la "automejora" y la "apertura al cambio". Esto se debe principalmente a factores culturales. En el grupo de los estudiantes se nota un mayor valor en la "auto-mejora" y la "apertura al cambio" mientras que en estos mismos valores los valores más bajos corresponden a los profesores.

\section{Conclusiones}

Puede concluirse que el instrumento de evaluación es válido en el contexto mexicano como ya se había corroborado en estudios previos realizados por Schwartz con datos de México. Aunque los 
estudios previos realizados en México únicamente fueron con estudiantes universitarios y profesores. En este sentido, es importante remarcar que no todas las culturas ni los individuos son iguales y que existen diferencias entre las escalas de valores como se demostró. Es decir que el enfoque presentado por Sasaki et al. (2013) es el adecuado para el estudio de las culturas a nivel internacional.

Los análisis factoriales practicados a los tres grupos de estudio (profesores, estudiantes y profesionistas) demuestran que los valores desarrollados por Schwartz son aplicables para ser medidos en México. Es necesario enfatizar que existen diferencias de estos tres grupos en cuanto a los constructos y que es necesario tener en cuenta que el valor "universalismo" no implica lo mismo para los tres grupos de estudio. Sin embargo a través del análisis se demuestra la aplicabilidad del instrumento.

Sin embargo, a través del análisis de los tres grupos se demuestra que los valores predominantes son la "trascendencia" y el valor de "conservar". Estos valores son opuestos a las necesidades de las empresas donde los cambios se dan de manera continua. El grupo que no presenta claramente la tendencia a estos valores es el de estudiantes.

Es importante destacar la relación entre los valores, la cultura y el comportamiento, pues nos indica el camino que una persona sigue en su actuar, esta relación debe ser estudiada y valorada en relación con otros elementos que nos conduzcan a aclarar cuáles serían los valores "óptimos" o preferidos y deben desarrollarse, así como conseguir la vía para alcanzar mejores resultados en el trabajo. Es bien sabido que dentro de cada una de las organizaciones existe, generalmente, un decálogo de valores hacia el trabajo. 
Maria Teresa de la Garza Carranza, Eugenio Guzmán Soria, Alfonso Cervantes Maldonado

\section{Referencias bibliográficas}

Burns, J. M. (1978). Leadership. New York: Harper \& Row.

Cayón, A., \& Pérez, E. (2008). Estructura de valores de Schwartz en el personal directivo universitario privado. Telos, 10, 3 , 403-417.

House, R., Javidan, M., Hanges, P., \& Dorfman, P. (2002). Understanding cultures and implicit leadership theories across the globe: An introduction to project GLOBE. Journal of world business, 37(1), 3-10.

Hanges, P. J., Javidan, M., Dorfman, P. W., \& Gupta, V. (2004). Culture, leadership and organizations (pp. 9-28). R. J. House (Ed.). Contemporary Leadership Theories: Enhancing the Understanding of the Complexity, Subjectivity and Dynamic of Leadership. California: Sage

Hofstede, G. (1980) Culture's consequences: International differences in work related values. California: Sage

Hofstede, G. (2001). Culture's consequences: Comparing values, behaviors, institutions and organizations across nations. California: Sage.

Jones, T., Felps, W., \& Gregory, B. (2007). Ethical theory and stakeholder related decisions. Academy of Management Review, 32(1), 137-157.

Kornblit, A. (1994). Vigencia del concepto de valor en las ciencias sociales. Buenos Aires: Instituto de Investigaciones Gino Germani, Universidad de Buenos Aires.

Maslow, A. (1954). Motivation and personality. New York: Harper.

Mizzau, L., \& Montanari, F. (2008). Cultural districts and the callenge of authenticity: the case of Piedmont, Italy. Journal of Economic Geography, 8(5), 651-673 

en cuanto a sus valores y sus implicaciones respecto al trabajo

Ralston, D. A., Holt, D. H., Terpstra, R. H., \& Kai-Cheng, Y. (2008). The impact of national culture and economic ideology on managerial work values: A study of the United States, Russia, Japan, and China. Journal of International Business Studies, 39(1), 8-26.

Ralston, D. A., Egri, C. P., Furrer, O., Kuo, M. H., Li, Y., Wangenheim, F., \& Weber, M. (2013). Societal-level versus individual-level predictions of ethical behavior: A 48-Society study of collectivism and Individualism. Journal of Business Ethics, 1-24.

Rokeach, M. (1973).The nature of human values. New York: Free Press.

Sasaki, I., \& Yoshikawa, K. (2014). Going beyond national cultures -Dynamic interaction between intra-national, regional and organizational realities. Journal of World Business, 49, 3, 455-464.

Schwartz, S. H., \& Bilsky, W. (1987). Toward a universal psychological structure of human values. Journal of Personality and Social Psychology, 53, 550-560.

Schwartz, S. H., \& Bilsky, W. (1990). Toward a theory of the universal content and structure of values. Journal of Personality and Social Psychology, 58, 878-891.

Schwartz, S. H. (1992). Universals in the content and structure of values: Theoretical advances and empirical tests in 20 countries. En M. P. Zana (Ed.). Advances in experimental social psychology (pp. 1-65). New York: Academic Press.

Schwartz, S. H. (1994). Are the universal aspects in the structure and contents of human values. Journal of Social, 50, 19-45.

Schwartz, S. H. (1996). Value priorities and behavior: Applying a theory of integrated value systems. In Seligman, Olson y 
María Teresa de la Garza Carranza, Eugenio Guzmán Soria, Alfonso Cervantes Maldonado

Zanna, [The Ontario Symposium, Vol. 8]. The psychology of values (pp. 1-24). New Jersey: Earlbaum.

Schwartz, S. H. (1997). Values and culture. En Munro, Carr y Schumaker (Ed.). Motivation and culture (pp. 69-84). New York: Routledge.

Schwartz, S. H. (2014). Rethinking the concept and measurement of societal culture in light of empirical findings. Journal of Cross-Cultural Psychology, 45(1), 5-13.

Williams, R. M. (1979). Change and stability in values and value systems: a sociological perspective. In M. Rockeach (Ed.). Understanding human values: Individual and societal (pp. 15-46). New York: Free Press.

\section{Dra. María Teresa de la Garza Carranza}

Ing. Industrial y de Sistemas por el Instituto Tecnológico y de Estudios Superiores de Monterrey, maestra en Ciencias en Liderazgo Educacional por la Florida International University; doctora en Ciencias con Especialidad en Ciencias Administrativas por el Instituto Politécnico Nacional. Ha publicado diversos artículos de investigación en revistas como Academy of International Business, Management Revue, Contaduría y Administración, etc. Ha publicado cuatro libros y ha contribuido en diversos capítulos de libros. Sus áreas de interés son: el comportamiento organizacional, la gestión 
educativa, las pequeñas empresas y la responsabilidad social corporativa. Actualmente es profesora e Investigadora del Instituto Tecnológico de Celaya (México).

Email: teresa.garza@itcelaya.edu.mx

\section{Eugenio Guzmán Soria}

Es profesor investigador del Postgrado en Administración en el Departamento de Ciencias Económico Administrativas del Instituto Tecnológico de Celaya (México). Es licenciado en Comercio Internacional de Productos Agropecuarios por la Universidad Autónoma Chapingo, con maestría y doctorado en Ciencias en Economía por el Colegio de postgraduados. Ha publicado varios capítulos y tiene 4 libros, varios capítulos del libro así como diversos artículos en revistas nacionales e internacionales de prestigio. Sus temas de investigación son: el análisis de precios, márgenes de comercialización de productos agrícolas, análisis econométrico de la oferta y demanda de productos, servicios e insumos (a través del uso de modelos de ecuaciones simultáneas); rentabilidad y competitividad (vía el algoritmo de la Matriz de Análisis de Política), óptimo técnico y económico de productos agropecuarios, entre otros.

Email: Eugenio.guzman@itcelaya.edu.mx 
Maria Teresa de la Garza Carranza, Eugenio Guzmán Soria, Alfonso Cervantes Maldonado

\section{Alfonso Cervantes Maldonado}

Realizó estudios de Ingeniería Industrial en el Instituto Tecnológico de León, maestría en Ingeniería de la Calidad en la Universidad Iberoamericana, maestría en Ingeniería Administrativa y Calidad en la Universidad de La Salle Bajío, actualmente cursa el doctorado en Administración y Estudios Organizacionales en la Universidad De La Salle Bajío. Sus temas de investigación están relacionados con actividades de ingeniería de procesos, gerencia de calidad, consultoría en sistemas de calidad y manufactura esbelta en organizaciones de manufactura; coordinador académico y profesor en instituciones educativas. Actualmente es profesor de la Universidad De La Salle Bajío (México).

Email: acervantes@delasalle.edu.mx

Recibido: 27/03/2014 Aprobado: 27/08/2014 